\title{
Eingabe an die Kantonsregierungen betreffend Ausführungsbestimmungen der Fristenlösung
}

\author{
N. Waldis
}

* AGEAS: Arbeitsgemeinschaft Evangelischer Aerzte der Schweiz; IANFP: Internationale Ärztevereinigung für Natürliche Familienplanung;

VKAS: Vereinigung Katholischer Ärzte der Schweiz.

Korrespondenz:

Dr. med. Niklaus Waldis

Case postale 6

CH-1630 Bulle
Die AGEAS, IANFP, Vereinigung Betroffenes Spital und VKAS* haben im Hinblick auf die Inkraftsetzung der vom Volk angenommenen Strafgesetzänderung über den Schwangerschaftsabbruch an sämtliche Kantonsregierungen geschrieben und um die Berücksichtigung ihrer Anliegen in 3 Punkten ersucht:

- dass die Beratung der Schwangeren durch qualifiziertes Personal und in lebensbejahender Art durchgeführt werde;

- dass die unterzeichnenden Organisationen bei der Ausgestaltung des Leitfadens miteinbezogen würden;

- dass die Möglichkeit der Verweigerung der Mitwirkung aus Gewissensgründen ohne irgendwelche Nachteile für die Betreffenden gewährleistet sei.

Von 18 Kantonen erhielten sie eine Empfangsbestätigung, von 12 weitergehende Informationen.

Drei Kantone (NE, SH, ZH) wiesen darauf hin, dass die Eingabe zu spät eingetroffen sei, so dass sie bei der Erarbeitung der Ausführungsbestimmungen und v.a. des Leitfadens nicht berücksichtigt werden konnte. Dies ist leider nur allzu wahr und u. a. darauf zurückzuführen, dass die betreffenden Organisationen ausschliesslich auf freiwillige Mitarbeiter zählen können.

\section{Ad 1}

4 Kantone sind darauf eingegangen: Obwalden erachtet die durch Art. 119 und 120 StGB vorgegebenen Minimalanforderungen als ausreichend. Im Kanton Bern hätten die für die Abtreibungsgutachtertätigkeit ermächtigten Ärzte seit 1980 eine langjährige Erfahrung, womit sich die Erarbeitung entsprechender Richtlinien erübrige. Im Kantonsspital Basel wird unter der Leitung von Prof. J. Bitzer ein Manual über die Grundlagen des Gesprächs und der Beratung erstellt. St. Gallen erwägt ein entsprechendes Weiterbildungsangebot für die betroffenen Ärzte.

\section{Ad 2}

Von 10 Kantonen (AG, AI, BL, BS, GL, OW, SG, TG, UR, ZG) erhielten wir ein Exemplar ihres Leitfadens. Diese Leitfäden basieren auf einem Modell, das eine Arbeitsgruppe von Vertretern der Vereinigung der Kantonsärzte und der Stiftung PLANeS (Schweizerische Stiftung für sexuelle und reproduktive Gesundheit) ausgearbeitet hat.

Im Text selber finden sich nur geringfügige Varianten. Jeder Kanton hat jedoch eine eigene Liste von Beratungs- und Hilfsstellen (3200 Adressen allein im Kanton Zürich), die aufzusuchen empfohlen wird.

Basel-Stadt hat uns freundlicherweise die Möglichkeit gegeben, vor dem Druck des Leitfadens unsere Verbesserungsvorschläge zum Aufbau oder zu Formulierungen schriftlich einzureichen.

Bei der Durchsicht der erhaltenen Leitfäden bez. unserer Forderungen weisen alle Kantone ausser Uri und Nidwalden auf lebensbejahende Beratungsstellen hin. Im Sitzungsprotokoll des Nidwaldner Landrates ist festgehalten, dass ganz bewusst keine solche Beratungsstelle erwähnt werden solle, obwohl verschiedene Organisationen schriftlich darum gebeten hatten.

Mit dem fehlenden Hinweis auf die Schweizerische Fachstelle für Adoption ist der Leitfaden von Baselland nicht gesetzeskonform.

Appenzell, Glarus, St. Gallen und Zug erwähnen zudem noch speziell die Pfarrämter als Entscheidungshilfe beim Erwägen einer Abtreibung.

Der Kanton Freiburg hat in seinem Ausführungsgesetz den Dienst für Familienplanung und Sexualinformation, der der Stiftung PLANeS nahesteht, zur öffentlichen Beratungsstelle erklärt und in den Rang einer Behörde erhoben. Von diesem Dienst erhielten die Freiburger Ärzte auf dem Papier der Sanitätsdirektion ein Informationsschreiben mit zwei Faltblättern. Auf einem davon stand folgende Devise: 
Il ne faudrait jamais que l'avortement se fasse sans des entretiens visant à faire sourdre de l'inconscient de chaque femme, tout ce que cet acte a d'enrichissant pour elle, son couple, son groupe, au lieu de le vivre comme un effacement technique, d'un incident technique, d'une vie génitale technique. (Citation Françoise Dolto)

Das Vermischen und Vertauschen der Begriffe von Gut und Schlecht in diesem Zitat zeigt, auf welch perfide Weise die Kultur des Todes Einzug in die Schweiz hält; das Abstimmungsergebnis vom 2. Juni 2002 belegt das Ausmass ihrer Verbreitung.

\section{Ad 3}

Bezüglich der Verweigerung aus Gewissensgründen erhielten wir folgende Antworten aus 5 Kantonen:

- In Basel-Stadt liegt die Entscheidungskompetenz bei der ärztlichen Leitung des Kantonsspitals. Dem Kantonsarzt sind keine Benachteiligungen bekannt.
- Im Kanton Bern sei eine Gewissensverweigerung gesetzlich erlaubt und in den grösseren Spitälern durchaus in der Praxis umsetzbar.

- In Neuenburg soll die Gewissensverweigerung prinzipiell respektiert werden. Eine Ethikkommission befasst sich mit diesem Thema und wird ihren Standpunkt demnächst bekanntgeben.

- In St. Gallen werde niemand gezwungen, gegen seinen Willen an einer Abtreibung mitzuwirken.

- Im Kanton Zug werden Bewilligungen, um Abtreibungen gesetzeskonform durchzuführen, nur an Ärzte erteilt, die ein Gesuch dazu einreichen. Wer generell keine Abtreibungen (mehr) durchführen will, kann auf die Bewilligung verzichten.

Theoretisch scheint die Frage der Gewissensverweigerung gut geregelt zu sein. In der Praxis sieht es leider anders aus; immer wieder hören wir von Medizinalpersonen, die einem massiven Mobbing ausgesetzt sind, weil sie sich weigern, an Abtreibungen mitzuwirken. Wo bleibt da die viel beschworene Offenheit und Toleranz der Abtreibungsbefürworter? 